of meat. With the buffaloes, as with the other animals, the Nuffield Unit, although engaged in a pure research project, is providing information which will be valuable to the management of the park in their work.

\section{Inhibitor Inhibited}

\section{from our Molecular Biology Correspondent}

THE trypsin inhibitors form an interesting class of lowmolecular weight proteins of remarkable specificity. Several plant and animal trypsin inhibitors have been studied, and quite recently the full amino-acid sequence of the bovine pancreatic inhibitor (often known as Kunitz and Northrop's basic inhibitor) has been de. termined. Like other animal trypsin inhibitors, this protein has three disulphide bonds, and their relation to the reaction with trypsin has been investigated by Kress and M. Laskowski, sen. (J. Biol. Chem., 242, 4925; 1967).

The sequence and position of the disulphide bonds were both reported earlier by Laskowski and his group. It now turns out that one of the disulphide bonds (between residues 14 and 38 ) can be reduced without damage to the other two-a reaction which has been paralleled in several other proteins. Again, as in one or two of these cases, the product retains its activity. If, however, the new sulphydryl groups are carboxymethylated, activity is lost and, indeed, the protein becomes prone to hydrolysis by trypsin. The partially reduced inactivator reforms the broken disulphide bond under the usual conditions of reoxidation in air; in its complexes with trypsin, however, the reoxidation is almost wholly prevented.

The drastic effect produced by the quite modest steric disturbance of introducing the two carboxymethyl groups is in interesting contrast to the observation of Chauvet and Acher (J. Biol. Chem., 242, 4247; 1967) that the four lysines of the inhibitor may be guanidinated without detriment to the activity. This reaction amounts to a small displacement of the charge by effectively lengthening the lysine side chain. The overall charge, however, must be maintained, for acetylation of the lysines inactivates the inhibitor completely. It is also interesting to note that whereas all five amino groups (the four lysines and the $N$-terminus) are reactive in the free state, one of them (lysine-15) is shielded in the complex with trypsin, and may therefore form part of the active site.

Both trypsin and the inhibitor are quite small molecules; the latter indeed has only 58 residues, and is therefore one of the smallest globular proteins (excluding from this definition the polypeptide antibiotics). This therefore provides one of the very simplest systems available for the study of specific interactions between proteins.

\section{Histone Synthesis in HeLa Cells \\ from our Cell Biolozy Correspondent}

THE discovery that histones are uniquely associated with DNA in cell nuclei and do not occur in the cytoplasm provoked the hypothesis that histones function as repressors of gene transcription and has led to attempts to establish a temporal relationship between histone and DNA synthesis. It now seems fairly certain that histones do have a role in genetic regulation, but whether DNA replication and histone synthe- sis are always synchronous is still an open question. No doubt much of the confusion about this relationship has arisen because different workers have used different methods to identify histones and synchronize cell populations and they have used different cell types. But Robbins and Borum (Proc. US Nat. Acad. Sci., $5 \%, 409 ; 1967)$ and Borum, Scharff and Robbins (ibid., $58,1977 ; 1967)$ seem to have established that, in HeLa cells at least, histone and DNA synthesis are synchronous and, more unexpectedly, that histones are synthesized in the cytoplasm.

Robbins and Borum used gel electrophoresis to identify two heterogeneous histone fractions in protein extracted from a synchronized population of HeLa cells with hydrochloric acid. With this method and using $\mathrm{C}^{14}$ lysine to label histones and $\mathrm{C}^{14}$ thymidine to label DNA, they found that lysine incorporation into histone is negligible during the $G_{1}$ phase of the cell cycle. But as the $S$ phase begins and DNA synthesis starts, lysine incorporation into hiscones increases. The incorporation of lysine into non-histone protein stays constant, however. Thus the synthesis of histones is uniquely synchronized with DNA synthesis.

Where are the histones synthesized? To answer this, synchronized cells were given a 2 minute pulse of $\mathrm{C}^{14}$ tryptophan, to label non-histone protein and $\mathrm{H}^{3}$ lysine, and then chased in a medium containing a large excess of unlabelled lysine and tryptophan. After 2 minutes incorporation, 80 per cent of the lysine was in the cytoplasm and during a 2-12 minute chase the amount of incorporated lysine in the nucleus increases by 33 per cent. Lysine rich protein is therefore being transported into the nucleus. Tryptophan labelled protein, however, shows no such transfer. The clear implication is that the histones are synthesized in cytoplasm, and if so it should be possible to detect cytoplasmic polysomes synthesizing histone.

Robbins and Borum analysed the distribution of incorporated lysine and tryptophan among HeLa polysomes from cells at $G_{1}$ and $S$ phases. At the $S$ phase in the cell cycle more small polysomes are present than at $G_{1}$ and a significantly greater percentage of the lysine is associated with the small polysomes than with large polysomes. Furthermore, when cytosine arabinose, an inhibitor which stops DNA and histone synthesis, is given during the $S$ phase, the lysine rich small polysomes disappear. They concluded that DNA and histone synthesis is synchronized and that the histones are made in the cytoplasm on small polysomes.

Borum, Scharff and Robbins, continuing the study, isolated the nascent polypeptides from the small cytoplasmic polysome fraction and found that they are lysine rich and migrate on gel electrophoresis like authentic histones from nuclei. A rapidly labelled RNA fraction isolated from the polysomes appears to be histone $m$ RNA. This RNA first appears at the beginning of the $S$ phase and its synthesis ceases about 2 hours before cell histone synthesis ceases at the end of the $S$ phase. The histone $m$ RNA has a short half life of $1-1.5$ hours.

These results indicate that the principal control of histone synthesis is at the level of gene transcription, but this does not, of course, exclude some fine control at the translational level. It will be very interesting to see whether or not all these conclusions are valid for other cell types. 